\title{
Cylinder Pressures in a Position Controlled System With Separate Meter-in and Meter-out
}

\author{
G. Rath and E. Zaev* \\ Department for Product Development, University of Leoben, Leoben, Austria \\ E-mail: gerhard.rath@unileoben.ac.at,emil.zaev@mf.edu.mk \\ *Faculty of Mechanical Engineering, Ss.Cyril and Methodius University in Skopje, Skopje, Macedonia
}

\begin{abstract}
Hydraulic servo system driven with separate meter-in and meter-out valves provide more flexibility with respect to energy efficiency and dynamic properties. In the current problem the operation of such a configuration involves to keep a desired position constant, while an external load force is applied. The position control cannot compensate for fast load fluctuations beyond a certain frequency limit. For this case it can be desired to adjust the static pressures in the cylinder chambers to defined values. The paper describes how to influence this pressures giving weights to the control signals for both moving directions for each valve. With the help of a simulation model the design results are verified. Higher pressure levels decrease the danger of cavitation and inhibit lowering the bulk modulus of the oil volume. The asymmetric control of the valves even lead to better energy efficiency during position control.
\end{abstract}

Keywords: Servo control, smismo, energy efficieny

\section{Introduction}

\subsection{Control of Hydraulic Actuators}

The main component for proportional velocity and position control of industrial or mobile hydraulic systems traditionally is a spool valve applied to each actuator. Analysis of the dynamic behaviour of such a system was done very early [1], and fast servo-valves were developed [2]. For a long time complex control algorithms were reserved for dedicated signal processors [3]. In the last decade programmable logic controllers (PLC) provided more and more computing power and enabled also working with non-linear models of hydraulic systems [4]. The control of the oil flow with valves causes energy loss due to pressure drop. Better efficiency can be obtained by including the pump in the control strategy, either without any additional valve or combining proportional valve with a load-sensing (LS). The dynamic behaviour of such a system was studied in [19].

The most important goal in the recent time is the development of energy efficient systems, which is a major topic for separate meter-in, separate meter-out control (SMISMO) hydraulics [8]. Development of robust sensors and new hydraulic designs for SMISMO enabled more sophisticated control of hydraulic drives, a higher degree of freedom is offered to control engineers. The analysis of the additional working modes provided by such a system can be found in [6] or [13]. It is even possible to recuperate energy, which may be important for heavy machinery [18].
SMISMO control may be combined with classical elements for hydraulic control, such as electronic load-sensing (ELS). Switching strategies for energy optimisation including multiple actuators in an ELS system are presented in [9]. Also over centre valves in SMISMO systems are considered [10].

Another important issue is vibration and oscillation damping. For large-scale machinery the energy efficiency is also a major topic for such applications [11]. With separate meter-in and meter-out exended with a crossport valve, active damping can be accomplished efficiently [12].

The separate meter-in and meter-out system increases the amount of variables to be influenced by electronic control, which requires to apply more sensors. Those are still expensive und not highly dependable. Methods to reduce the amount of sensors are also a research topic, for example in [5].

Sometimes it is desired to simplify the control strategy in order to influence certain variables of the hydraulic and mechanical system more specifically. For example, a decoupled control of variables in a multiple-input/multiple output (MIMO) system, can be found in [7]. The work presented in this paper describes how to influence the chamber pressures during constant position control without the need of an additional control loop.

Most work about separate meter-in and meter-out is done on how to control the motion in an energy efficient manner. Here in contrast a situation is investigated, where the position is regulated to be constant. A solution for a two-valve configu- 
ration in a position control application is shown, that allows to influence the pressures within certain limits. Beside for position, no additional sensors are used.

\subsection{System Description}

The hydraulic machine considered here is equipped with a milling head driven with an electric motor. Motion and positioning of the head is done with two hydraulic axes for two degrees-of-freedom. Each of them is controlled with two proportional valves in a SMISMO configuration. When one axis is moving for the milling operation, the other axis stands still and is subjected to load fluctuations caused by the milling process. The purpose of the regulator is to keep the passive axis in a constant position. The goal of the design process proposed here is to optimise the pressure levels in the cylinder chambers so, that maximum force impacts can be caught without the occurrence of cavitation. The hydraulic power supply is a load-sensing system. Since the active cutting axis usually needs the full pressure provided by the pump, the passive axis, which holds its position, is not included in the loadsensing loop. Consequently the maximal, or at least a high pressure level is available for position control. The most im-

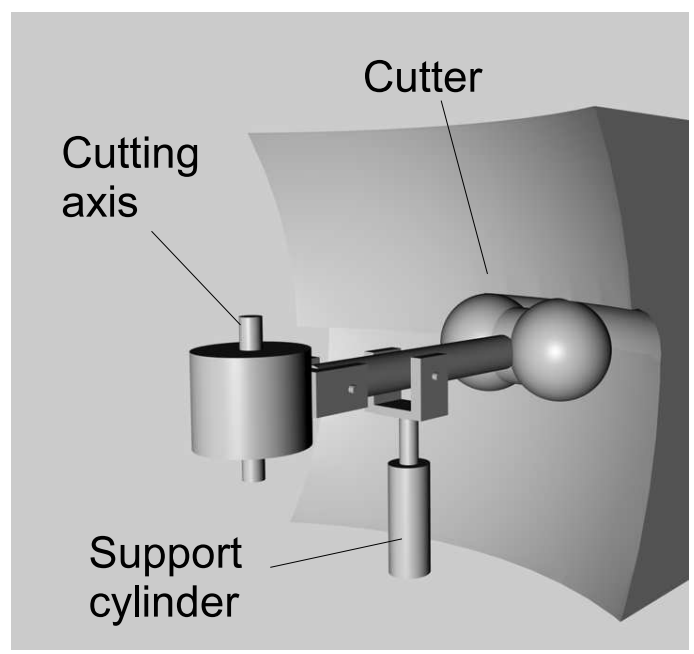

Figure 1: Hydraulic scheme

portant components for the control of the passive hydraulic axis, which is considered, are shown in Fig. 2. It is assumed for the investigations, that the pump provides a constant high pressure during the milling process for the active axis. Two four-land-four-way spool valves are applied to establish separate meter-in and meter-out control. Some auxiliary hydraulic elements of minor relevance are not sketched in Fig. 2.

\subsection{Problem Description}

The actual system is equipped with separate meter-in and meter-out control to allow energy efficient control of motion during several working situations. One strategy for example is to control speed with the meter-in valve while keeping the pressures at a reasonable level with the meter-out valve to avoid high power losses on the one hand, and to avoid cavitation in the case of overrunning load on the other [6].

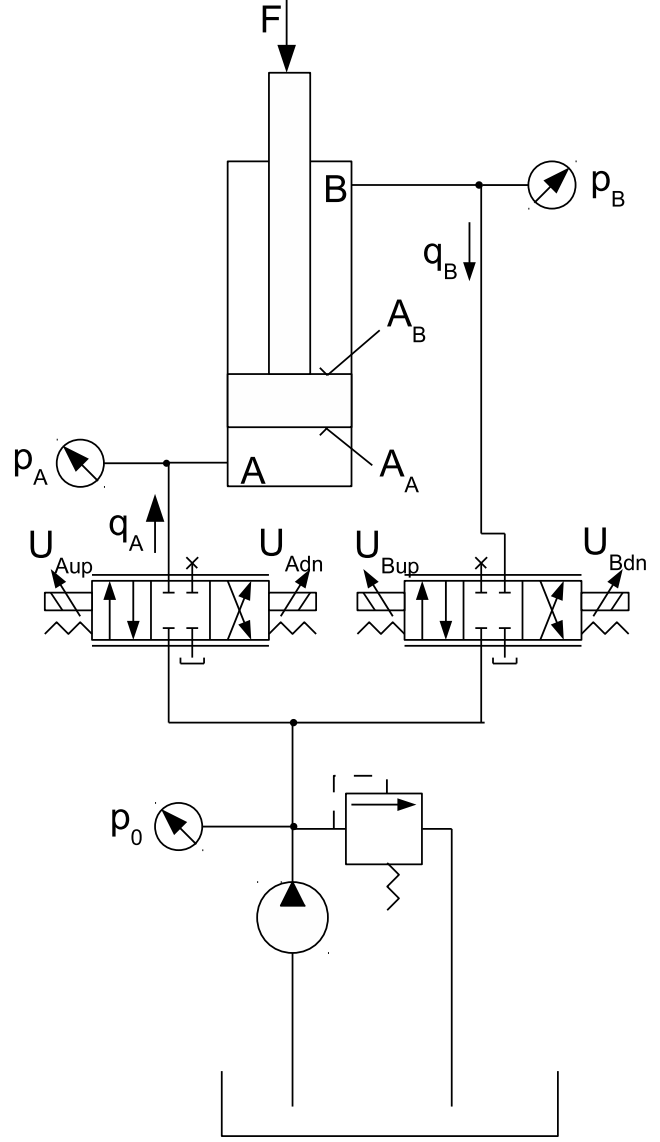

Figure 2: Hydraulic scheme

Cutting operation of the system investigated here requires an additional operating mode, where the support cylinder should not move, but hold its position subject to external forces. For this purpose, a position control loop is required. Now the separate meter-in and meter-out system allows an additional degree of freedom, which is utilised in this work. It is investigated how to improve the performance of position control without adding further sensors or control loops.

\section{Electronic Control}

The scheme for the electronic position regulator of the cylinder is displayed in Fig. 3. This control loop will ensure,

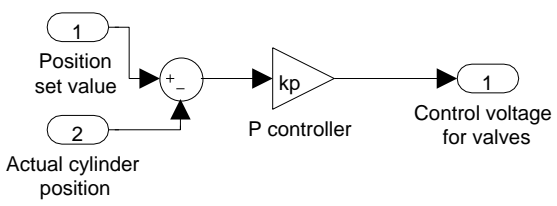

Figure 3: Basic position control

that the mean value of the position is held constant independently from the magnitude of the load force generated by the cutting process. A simple proportional $(\mathrm{P})$ controller is assumed here. From the authors' experience, a more sophisticated algorithm, see [17], does not influence the result of the considerations made here. The control error is multiplied with the 
gain and provided as output signal. If both the valves are driven with this signal and have identical characteristics, the result is equivalent to the control of the cylinder with a single servo or proportional valve.

The individual metering valves provide an additional degree of freedom that is utilised here. A factor $\lambda$ is introduced that provokes asymmetric control voltages for the valves dependent on the moving direction. These motions around a desired setvalue of the cylinder displacement are caused by the external load force and not due to a change of the set value (regulator case). Fig. 4 shows the function block inserted between the position controller and the outputs for the proportional valves.

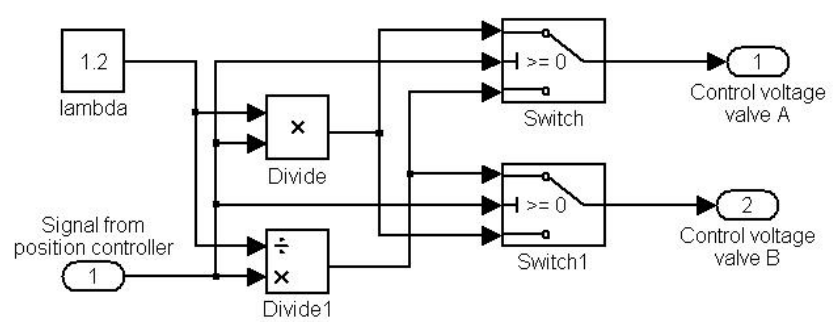

Figure 4: Signal flow for pressure adjustment

The effect of this interception is to introduce a virtual imbalance of the proportional valves. The effect of the electronic imbalance can be understood easier studying a mechanical equivalent in Fig. 5. A thick arrow represents an increased orifice multiplied with the factor $\lambda$ greater than one. Small

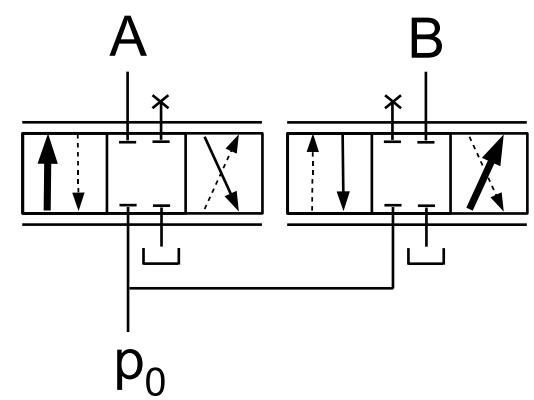

Figure 5: Machanical equivalent for electronic pressure adjustment

arrows indicate a reduced opening by multiplication with $\frac{1}{\lambda}$. Dotted arrows are not active in this configuration. Looking at this model makes clear that the paths to the supply pressure are wider than those to the tank. Hence higher pressure levels are to be expected in the cylinder chambers.

The advantage of the electronic imbalance over a fixed mechanical asymmetry is that a change for other working conditions is easy. For example, if the axis should move over a distance, a different concept for individual metering can be used to optimise the energy efficiency.

\section{Pressure Levels}

The procedure how to calculate the pressure levels in the cylinder chambers of a hydraulic system under electronic control for a constant piston displacement controlled with a single servo or proportional valve was already presented and verified in [14]. Here the scope is extended to separate meterin and meter-out valves including the method according to Fig. 4 for introducing an imbalance. The scheme with a factor $\lambda$ was chosen in order to get a simple result in the following procedure.

An external force is applied that fluctuates. As intended, position control will hold the mean values of the oil volumes constant. From this follows, that the mean values of the oil flows to and from of the cylinder chambers to zero. The output of the proportional controller follows the control error with the factor $k_{p}$. Normally the integrating behaviour of the cylinder forces the mean value of the control error to zero. Since the oil flows to and from the cylinder depend on the pressures and hence are different for each chamber, the control error has to assume an offset (Fig. 6). This offset,

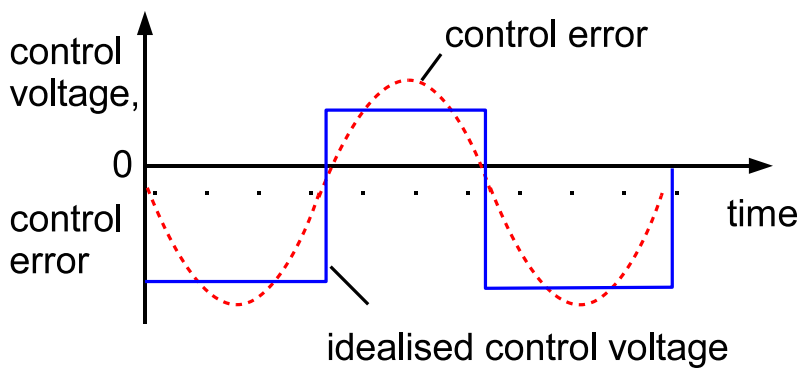

Figure 6: Small oscillations of displacement and controller output

which will be very small in a typical system, can be used to find conditions for the pressures in the cylinder. Approximating now the controller output with square oscillations yields two levels $U_{u} p$ and $U_{d} n$.

These oscillations may have the natural frequency of the system or the frequency of the external force. Oil flows are small, consequently pressures do not change much. At this point it should be mentioned that the law of continuity of the oil flows does not apply here, since the flows are mainly contributed by charging and discharging the chamber capacities.

A cylinder is moving 'up' when an oil flow $q_{A}$ enters chamber A (Fig. 2). The valve connected to A is supplied with a pressure $p_{0}$ from the pump. Chamber B is opened by a separately controlled valve to the tank. During motion 'down', chamber $\mathrm{B}$ is connected with the supply and A with the tank. In the first step, both valves accept the same control voltage $U$ from the position controller in Fig. 3.

For a small motion down we get

$$
\begin{aligned}
& q_{B d n}=U_{B d n} k_{B} \sqrt{p_{0}-p_{B}} \\
& q_{A d n}=U_{A d n} k_{A} \sqrt{p_{A}} .
\end{aligned}
$$


For a small motion up we get

$$
\begin{aligned}
& q_{\text {Bup }}=U_{\text {Bup }} k_{B} \sqrt{p_{B}} \\
& q_{\text {Aup }}=U_{\text {Aup }} k_{A} \sqrt{p_{0}-p_{A}} .
\end{aligned}
$$

What is important here, is the fact, that the position will be held with the exception of a small offset. This is the desired behaviour of the control loop. From this follows, that the oil flow coming out of one chamber must be the same going in during the next half period of the oscillation, which means

$$
\begin{aligned}
& q_{\text {Bdn }}=q_{\text {Bup }} \\
& q_{\text {Adn }}=q_{\text {Aup }} .
\end{aligned}
$$

And further, with Equ. 1 and 2, follows

$$
\begin{aligned}
& U_{B d n} k_{B} \sqrt{p_{0}-p_{B}}=U_{B u p} k_{B} \sqrt{p_{B}} \\
& U_{A d n} k_{A} \sqrt{p_{A}}=U_{A u p} k_{A} \sqrt{p_{0}-p_{A}}
\end{aligned}
$$

and

$$
\begin{aligned}
& \frac{U_{B d n}}{U_{B u p}}=\frac{\sqrt{p_{B}}}{\sqrt{p_{0}-p_{B}}} \\
& \frac{U_{A d n}}{U_{A u p}}=\frac{\sqrt{p_{0}-p_{A}}}{\sqrt{p_{A}}} .
\end{aligned}
$$

As depicted in Fig. 6, the position controller is assumed to deliver two discrete output levels, a positive voltage $U_{u p}$ and a negative voltage $U_{d n}$. Now the circuit in Fig. 4 is intercepted between the controller and the valves. Positive and negative control output values are weighted differently for both valves in the following way

$$
\begin{aligned}
& \frac{U_{B d n}}{U_{\text {Bup }}}=\frac{\lambda \cdot U_{d n}}{\frac{1}{\lambda} \cdot U_{u p}}=\lambda^{2} \frac{U_{d n}}{U_{u p}} \\
& \frac{U_{A d n}}{U_{A u p}}=\frac{\frac{1}{\lambda} \cdot U_{d n}}{\lambda \cdot U_{u p}}=\frac{1}{\lambda^{2}} \frac{U_{d n}}{U_{u p}},
\end{aligned}
$$

hence Equ. 5 turns to

$$
\begin{aligned}
\lambda^{2} \frac{U_{d n}}{U_{u p}} & =\frac{\sqrt{p_{B}}}{\sqrt{p_{0}-p_{B}}} \\
\frac{1}{\lambda^{2}} \frac{U_{d n}}{U_{u p}} & =\frac{\sqrt{p_{0}-p_{A}}}{\sqrt{p_{A}}}
\end{aligned}
$$

from which we get

$$
\frac{p_{B}}{p_{0}-p_{B}}=\lambda^{8} \frac{p_{0}-p_{A}}{p_{A}} .
$$

Pressures A and B depend on the mean value of the cylinder load

$$
F=p_{A} A_{A}-p_{B} A_{B} .
$$

Consequently pressure $p_{B}$ becomes

$$
p_{B}=p_{A} \frac{1}{\eta}-F^{\prime}
$$

with $F^{\prime}=\frac{F}{A_{B}}$ and $\eta=\frac{A_{B}}{A_{A}}$. Finally follows

$$
\begin{aligned}
& p_{A}^{2} \frac{1}{\eta}\left(1-\lambda^{8}\right)+p_{A}\left[\lambda^{8} p_{0}\left(1+\frac{1}{\eta}\right)-F^{\prime}\left(1-\lambda^{8}\right)\right]- \\
& -\lambda^{8} p_{0}\left(F^{\prime}+p_{0}\right)=0 .
\end{aligned}
$$

This is a quadratic equation that delivers the solution for $p_{A}$. Pressure $p_{B}$ follows from Equ. 10. Of course Equ. 11 has two solutions. For $\lambda<1$ one solution has negative pressures, which is not possible for the physical system. For $\lambda>1$ one solution exists with all pressures higher than the supply, which is also not possible. Sign information was lost after squaring Equ. 7. For $\lambda=1$ Equ. 11 turns into a linear equation, a case, which was already treated and verified by measurements in [14].

Consequently there exists only one solution for a physical system. It is the result with the positive sign in the solution formula for the quadratic equation. The pressure levels during a constant position control dependent on the load force can be found in Fig. 7. An arbitrary cylinder with equal areas is chosen. It can be seen that by selecting $\lambda$ the pressures

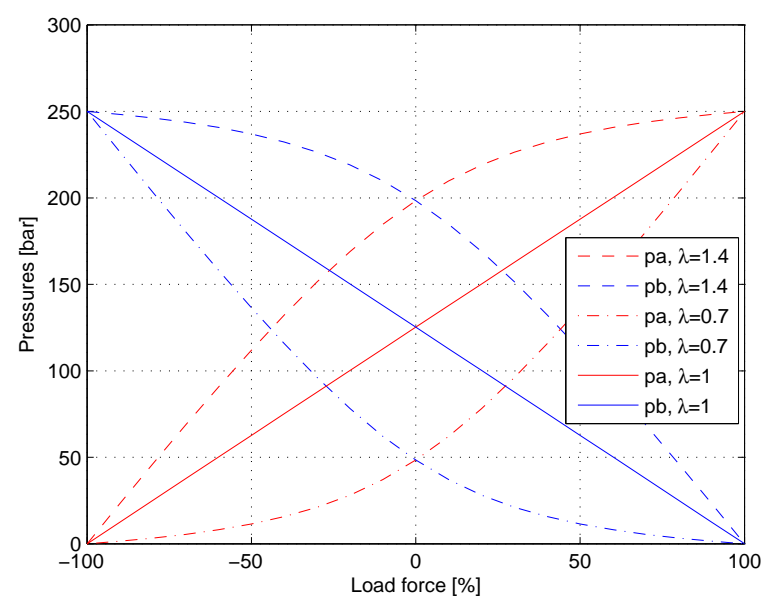

Figure 7: Quiescent pressures dependend on load force

in the cylinder may be varied. With the imbalance factor $\lambda$ greater than one the levels can be drawn towards the supply pressure, or vice versa. Of course at positive or negative force maximum, there is no chance to influence the pressures.

Remarkable is, that different characteristics of the valves, i.e. the ratio of $k_{A} / k_{B}$, does not contribute to the pressure levels. Of course there is an influence when the cylinder is moving at a desired speed, but this case is not a topic in this considerations.

Fig. 4 represents the implementation of Equ. 6, and Equ. 11 evaluates the resulting pressure levels dependent on the mean external load force.

\section{Simulation Model}

In order to verify the pressure predictions in the previous section and to study the behaviour in various situations, a simulation example is carried out here. The properties of the system are studied applying load forces to the model, first following an idealised step function. Then a force progression is chosen that is typical for the physical system under investigation.

The data that are used for the simulation model are listed in Tab. 1. The fully opened orifices of the valve spool deliver 


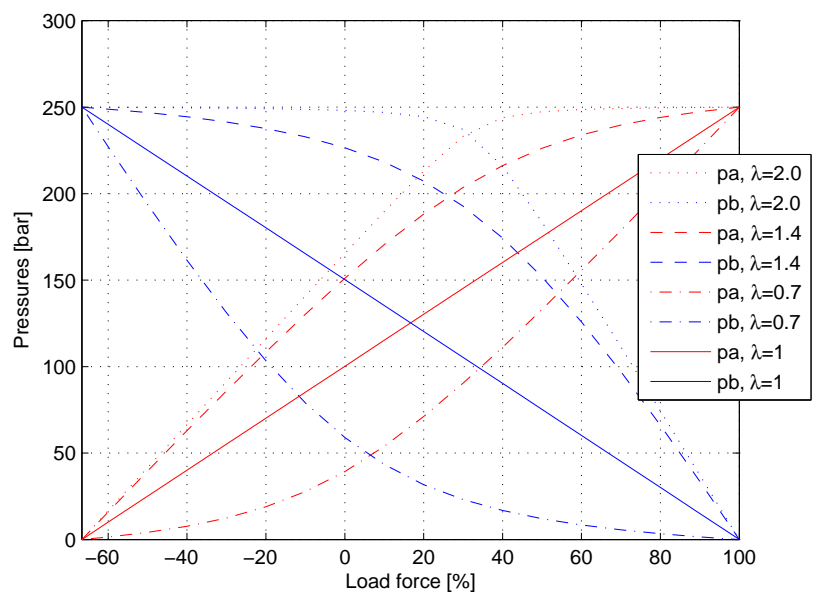

Figure 8: Pressures for eta $=0.7$

$1701 \mathrm{~min}^{-1}$ at a pressure difference of 70 bar.

\begin{tabular}{lll}
\hline Designation & Denotation & Value \\
\hline$A_{A}$ & Area piston side & $0.06 \mathrm{~m}^{2}$ \\
$A_{B}$ & Area rod side & $0.04 \mathrm{~m}^{2}$ \\
$p_{0}$ & Supply pressure & $250 \cdot 10^{5} \mathrm{~Pa}$ \\
$k_{A}$ & Valve A characteristic & $1.07 \cdot 10^{-6}$ \\
& & $\mathrm{~m}^{3} \mathrm{~s}^{-1} \mathrm{~V}^{-1} \mathrm{~Pa}^{-1 / 2}$ \\
$k_{B}$ & Valve B characteristic & $1.07 \cdot 10^{-6}$ \\
& & $\mathrm{~m}^{3} \mathrm{~s}^{-1} \mathrm{~V}^{-1} \mathrm{~Pa}^{-1 / 2}$ \\
$\eta$ & Ratio $A_{B} / A_{A}$ & 0.7 \\
$m$ & Cutter head mass & $11500 \mathrm{~kg}^{-1}$ \\
$l$ & Lever ratio & 5 \\
$C_{A}, C_{B}$ & Hydr. capacities & $8.44 \cdot 10^{-12}$ \\
& & $\mathrm{~m}^{3} \mathrm{~Pa}^{-1}$ \\
$U_{A d n}, U_{A d n}$ & Valve A control voltage & $\max ^{\prime} \pm 1 \mathrm{~V}$ \\
& dependent on direction & \\
$U_{B d n}, U_{B d n}$ & Valve B control voltage & $\max . \pm 1 \mathrm{~V}$ \\
& dependent on direction & \\
\hline
\end{tabular}

Table 1: Data used for modelling

The pressure levels for this system obtained with Equs. 11 and 10 are shown in Fig. 8. Numerical simulation will yield the same results. Simulation experiments were carried out with Matlab $^{\mathrm{TM}} /$ Simulink $^{\mathrm{TM}}$. After starting the simulation, the system needs some time to settle, before the actual experiment can begin. This time is not displayed in the diagrams of the following simulations.

\subsection{Response to Load Force Step}

After creating a simulation model, a step response allows to verify the solution of Equ. 11 and to study some basic behaviour. In this experiment, load steps of $\pm 40 \%( \pm 600 \mathrm{kN})$ are applied. Starting with load zero, the positive step is applied at time $t=5 \mathrm{~s}$ and taken back at $t=10 \mathrm{~s}$. The negative pulse follows at $t=20 \mathrm{~s}$ until $t=25 \mathrm{~s}$. Fig. 9 shows the results for $\lambda=1.0$, Fig. 10 for $\lambda=1.4$.

Increasing $\lambda$ shifts the pressure levels towards the supply pressure, as seen also in Fig. 8. Sudden force changes can make a pressure exceed the supply level. This is acceptable until a certain limit, an obligate relieve valve will protect the system from overpressure. On the other hand, a pressure falling to zero, like in Fig. 9 without imbalance, is not acceptable since cavitation occurs. This is the main advantage of introducing an imbalance with $\lambda$ greater than one.

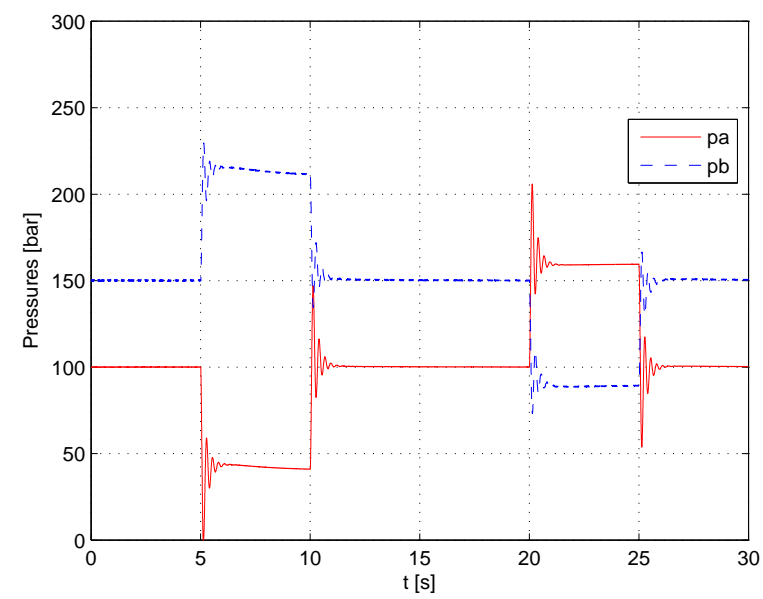

Figure 9: Pressure changes due to force steps, $\lambda=1.0$



Figure 10: Pressure changes due to force steps, $\lambda=1.4$

\subsection{Response to Forces During Operation}

The hydraulic system considered here is a positioning system for a milling device with external electrical drive. One hydraulic axis is used to move the cutter (active axis). The second cylinder, which is the item of interest here, is used to hold one coordinate of the tool constant (passive axis). In such a configuration, the cutting force is primarily generated by the external cutter drive, and the hydraulic cylinder has to hold its position constant. Hence a position regulator is required like the one shown in Fig. 3. 
While in the previous section the response to sudden load changes (step or Heaviside function) was studied, the behaviour under realistic conditions is of interest here. For this purpose, force data are used that are to be expected in practical operation, see Fig. 11. The maximal value displayed on the y-axis is the maximal force the cylinder can exert at the operating pressure of 250 bar. Fig. 12 shows the pressures

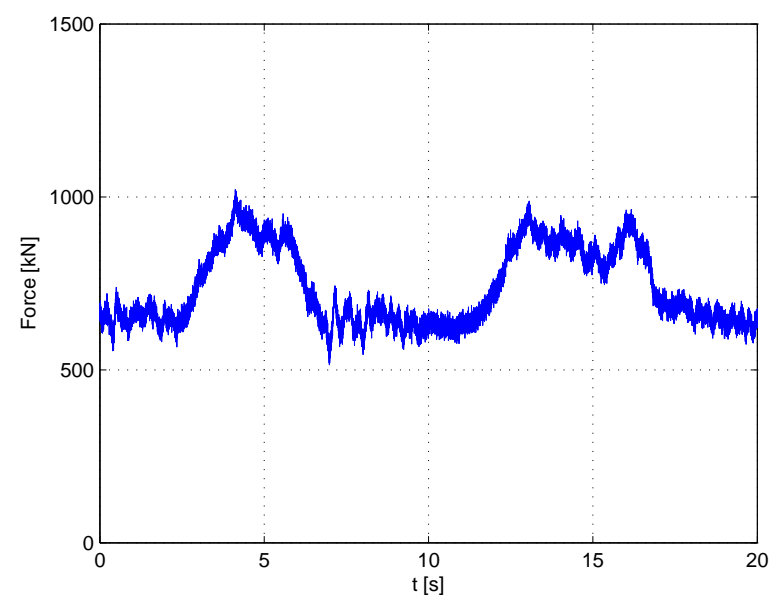

Figure 11: Force applied to the mass

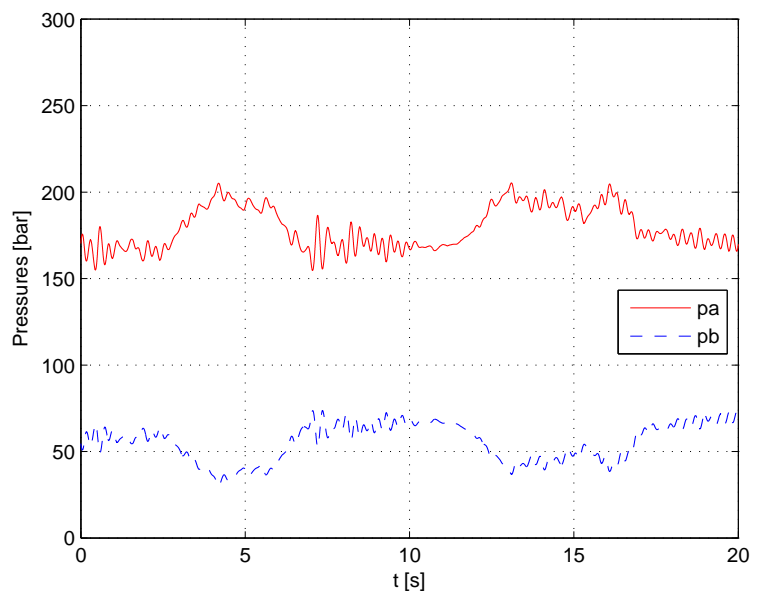

Figure 12: Cylinder pressures with $\lambda=1.0$

from simulation experiment with symmetrical excitation of the meter-in and the meter-out valve, which means, $\lambda$ is set to one in the circuit in Fig. 4. Alternatively, Fig. 13 displays the results for $\lambda=1.4$. As expected, the pressure levels are higher.

\subsection{Advantages}

Low pressure values decrease the bulk modulus of the oil due to entrained air [15] and [22]. Measurements in [16] show that particularly values below 50 bar are of disadvantage. Datasheets of rubber hoses also show higher compliance at low pressures. Furthermore, the danger of cavitation due to force peaks is increased.

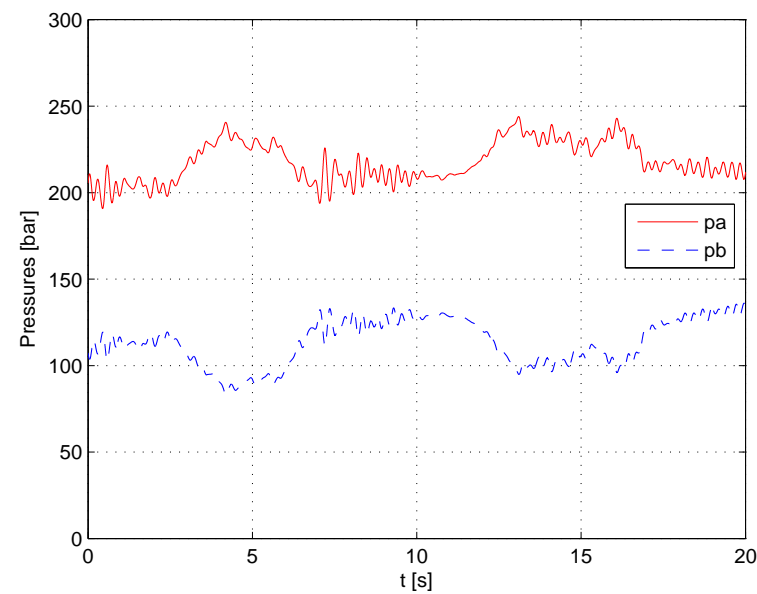

Figure 13: Cylinder pressures with $\lambda=1.4$

Using a control loop with an artificial imbalance $\lambda$ increases the pressure levels and improve the situation in this special operating mode, where the position should be held constant at a desired value.

\subsection{Control Gain and Energy Efficiency}

A position controller was a precondition for evaluating the pressure levels ending up in Equ. 11. Looking at this equation, it is remarkable that the gain has no influence to the long-term quiescent pressure levels. Engineers might tune the controller in order to optimise some property of the closed loop, for example as described in [20] for hydraulic systems. In the case of heavy machinery the energy efficiency is of primary interest to design the controller [21].

To keep the desired position, the controller will open the valves and thus consume oil from the high-pressure supply. The power related to the control action is not to be neglected and must be regarded in the design. Reducing the gain will lower the system's resistance against position change and lower the power consumption. Mean value of the position are kept as well as the steady-state pressure levels. Hence control gain is an important design parameter with respect to power requirement. As an extreme value, a gain of zero is equivalent to closed cylinder chambers, but then position and pressures will drift away due to leakage and possible opening of protective pressure relief valves. Consequently the choice of the gain is a trade-off between loss of energy and position accuracy.

Fig. 14 displays the power required during the operation subject to the forces in Fig. 11 and shows the importance of a proper gain design. Simultaneously the diagram shows that increasing $\lambda$ can improve the efficiency. When pressure levels in the cylinder are higher, the loss due to incoming oil is expected to be lower.

Stating that a low gain is better concerning power consumption, the influence of the gain to the loop behaviour must be investigated. Assuming, that the cylinder should change its position, the time behaviour usually is a measure for the quality of the design. Fig. 15 shows the time required for a po- 


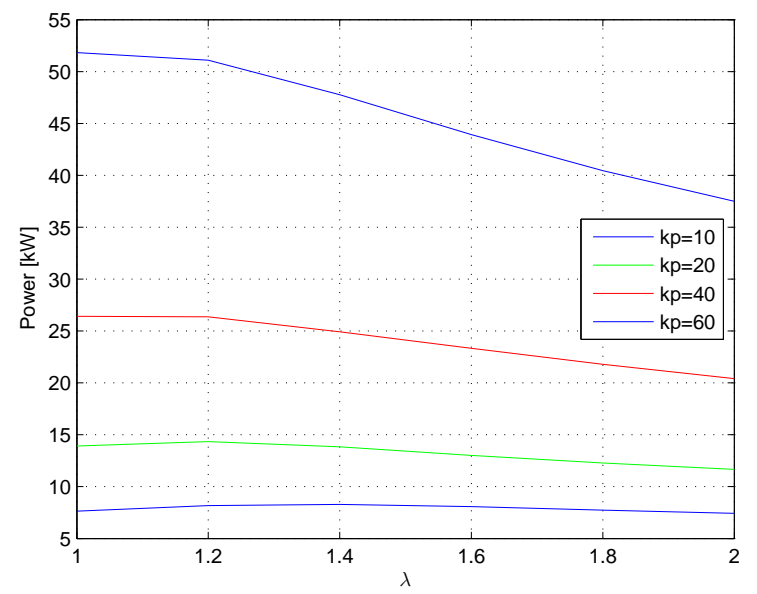

Figure 14: Power drawn from supply by position regulator

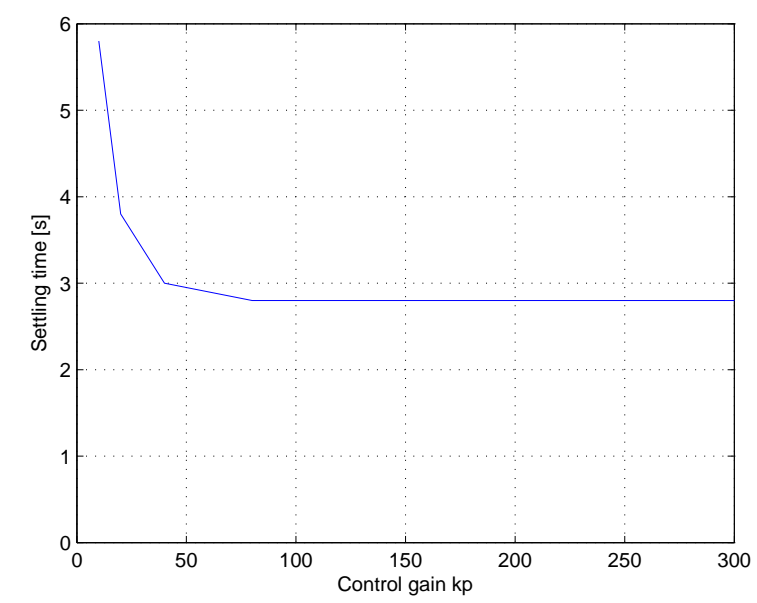

Figure 15: Settling time for a large motion of $100 \mathrm{~mm}$

sition change of $100 \mathrm{~mm}$ in the simulated system. From this diagram follows that the system is explicitly non-linear. Nevertheless a proper value for the gain can be found, which is as low as possible but does not increase the time constant. Further experiments that are not included in this paper show that the choice of $\lambda$ has almost no influence to this time behaviour. Motions with the position controller active will be only useful for small changes. For larger distances of course one of the strategies utilising the advantage of separate meter-in and meter-out control is to be preferred.

When the aim is to hold a constant position, the remaining question is how the design influences the position control error during operation. Fig. 16 shows the results from experiments with the simulation model. The root-mean-square (RMS) control error is defined as

$$
e_{r m s}=\lim _{T \rightarrow \infty} \sqrt{\frac{1}{T} \int_{0}^{T} e^{2}(t) \mathrm{d} t}
$$

where the time $T$ was 20 seconds in the experiment. Fig. 16

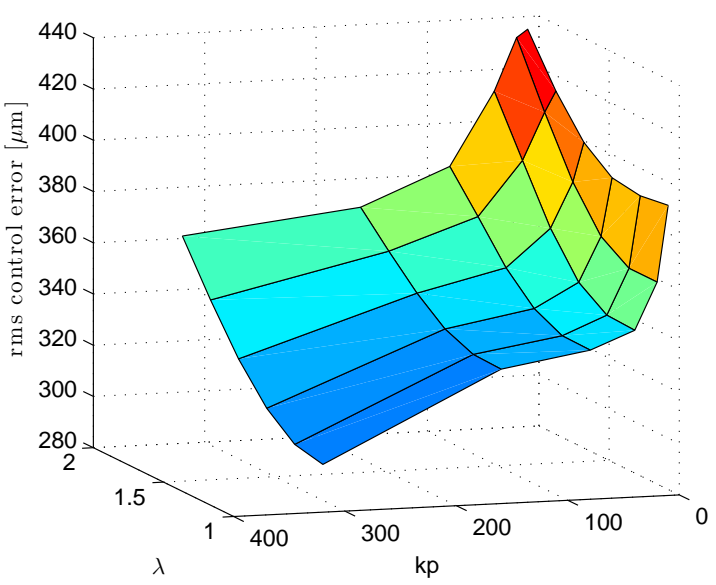

Figure 16: Root-mean-square control error

also shows a slight influence of the factor $\lambda$.

Furthermore it can be seen, that the RMS value starts rising at nearly the same low gain values, at which the settling time in the previous experiment was increased. Both design approaches lead to the similar results.

This relationship is not compulsory. In the first case the valves are opened fully and the systems gets non-linear. In the regulator mode, where the RMS errors are taken, the elongations and valve control voltages are very small. It is recommended to repeat both design steps for a specific machine.

\section{Conclusion}

A hydraulic cylinder, which supports a cutting tool head and is subject to high load fluctuations, is controlled with two individual metering valves. In order to hold the position constant a proportional regulator is used. Separate meterin and meter-out concept usually is applied to improve the energy efficiency during motion. In this work the additional degree of freedom is used to influence the pressure levels at a constant piston displacement during the cutting operation. With the help of electronic control an artificial imbalance is induced to flow control valves.

The effect is modelled with a single factor that allows to calculate the pressure levels in the cylinder in the closed position control loop. The loop is tested in a simulation model first with force steps, then with a set of force data that are typical for practical operation.

Tuning the imbalance factor allows to gain higher pressure levels in the cylinder chambers and hence to be less susceptible to cavitations and to avoid lowering the effective bulk modulus at low pressures. The imbalance slightly increases the control error and has no effect to the time behaviour of the loop. Since the proportional gain of the position controller has a great influence to the power consumption, the design of this gain is discussed.

As an additional effect it turns out that the imbalance also improves the energy efficiency of the system. The proposed 
method requires no additional sensor. Furthermore it is an open loop method, so the stability of the position control loop or possible other control loops is not influenced.

\section{Nomenclature}

\begin{tabular}{lll}
\hline Designation & Denotation & Unit \\
\hline$A_{A}$ & Area piston side & $\mathrm{m}^{2}$ \\
$A_{B}$ & Area rod side & $\mathrm{m}^{2}$ \\
$p_{0}$ & Supply pressure & $\mathrm{Pa}$ \\
$k_{A}$ & Valve A characteristic & $\mathrm{m}^{3} \mathrm{~s}^{-1} \mathrm{~V}^{-1} \mathrm{~Pa}^{-1 / 2}$ \\
$k_{B}$ & Valve B characteristic & $\mathrm{m}^{3} \mathrm{~s}^{-1} \mathrm{~V}^{-1} \mathrm{~Pa}^{-1 / 2}$ \\
$\lambda$ & Factor of signal unba- & \\
& lance for pressure adjust- & \\
& ment & \\
$\eta$ & Ratio $A_{B} / A_{A}$ & \\
$U_{d n}, U_{u p}$ & Controller output de- & $\mathrm{V}$ \\
$U_{A d n}, U_{A d n}$ & pendent on direction & \\
& Valve A control voltage & $\mathrm{V}$ \\
$U_{B d n}, U_{B d n}$ & dependent on direction & \\
& Valve B control voltage & $\mathrm{V}$ \\
& dependent on direction & \\
\hline
\end{tabular}

\section{Acknowledgement}

The authors would like to thank the engineers of the Sandvik Mining and Construction GmbH in Zeltweg, Austria, for inspiration and fruitful discussions and further Prof. Paul O'Leary, head of the Chair of Automation at the University of Leoben, for enabling this project.

\section{References}

[1] H E Merritt. Hydraulic Control Systems. John Wileys and Sons, Cincinnati, Ohio, 1967. ISBN 0-471-596175 .

[2] W. J. Thayer. Transfer functions for moog servovalves. In Technical Bulletin 103. Moog Inc., East Aurora, NY, 1965.

[3] R. Poley. Dsp control of electro-hydraulic servo actuators. In Application Report SPRAA76. Texas Instruments, 2005.

[4] M. Jelali and A. Kroll. Hydraulic Servo-systems: Modeling, Identification and Control. Springer, London, 2003.

[5] C.C. DeBoer and B. Yao. Energy-efficient motion control of a digital hydraulic joint actuator. In Proceedings of IMECE'01 2001 ASME International Mechanical Engineering Congress and Exposition, pages DSC2B-3, New York, USA, 2001. Japan Fluid Power System Society.

[6] K.A. Tabor. Velocity based method of controlling an electrohydraulic proportional control valve. United States patent application No. 20040055453, 2004.
[7] B.K. Nielsen. Controller Development for a Separate Meter-in Separate Meter-out Fluid Power Valve for Mobile Applications. PhD thesis, Institute of Energy Technology, Aalborg University, Aalborg, Denmark, 2005.

[8] B. Eriksson. Mobile Fluid Power Systems Design with a Focus on Energy Efficiency. PhD thesis, Institute of Technology, Linköping University, Linköping, Sweden, 2010.

[9] A.H. Hansen, H.C. Pedersen, T.O. Andersen, and L. Wachmann. Design of energy efficient smismo-els control strategies. In 2011 International Conference on Fluid Power and Mechatronics (FPM), pages 522-527, Aug. 2011.

[10] H.C. Pedersen, T.O. Andersen, Hansen R.H., and S. Stubkier. Investigation of separate meter-in separate meter-out control strategies for systems with over centre valves. In Proc. of the ASME Symposium on Fluid Power and Motion Control, FPMC 2010. American Society of Mechanical Engineers, 2010.

[11] B. Eggers, R. Rahmfeld, and M. Ivantysynova. An energetic comparison between valveless and valve controlled active vibration damping for off-road vehicles. In Proceedings of the 6th JFPS International Symposium on Fluid Power, pages 276-283, Tsukuba, Japan, 2005. Japan Fluid Power System Society.

[12] E. Zaev. 'Hardware-In-The-Loop' for real-time simulation of complex mechanical systems and their control. $\mathrm{PhD}$ thesis, Faculty of Mechanical Engineering, SS. Cyril and Methodius University, Skopje, Macedonia, 2013.

[13] A. Shenouda. Quasi-Static Hydraulic Control Systems and Energy Savings Potential Using Independent Metering Four-Valve Assembly. PhD thesis, Woodruff School of Mechanical Engineering, Georgia Institute of Technology, Georgia, 2006.

[14] G. Rath. Dynamics of high precision position controlled hydraulic cylinder subjected to elastic load. In K.T. Koskinen and M. Vilenius, editors, The Eighth Scandinavian International Conference on Fluid Power, Proceedings, pages 383-396, Tampere, Finland, 2003.

[15] W. Backé and H. Murrenhof. Grundlagen der ölhydraulik. Lecture notes, RWTH Aachen, Germany, 1994.

[16] R. Forsberg. Cut profile accuracy of roadheaders: Influence of different components and measures for improvements. Master's thesis, KTH Machine Design, Stockholm, Sweden, 2005.

[17] G.F. Franklin, M.L. Workman, and D. Powell. Digital Control of Dynamic Systems. Addison-Wesley Longman Publishing Co., Inc., Boston, MA, USA, 3rd edition, 1997.

[18] K. Heybroek. Saving Energy in Construction Machinery using Displacement Control Hydraulics, Concept Realization and Validation. $\mathrm{PhD}$ thesis, Institute of Technology, Linköping University, Linköping, Sweden, 2008. 
[19] P. Krus. On Load Sensing Fluid Power Systems, With Special Reference to Dynamic Properties and Control Aspects. PhD thesis, Department of Mechanical Engineering, Linköping University, Linköping, Sweden, 1988.

[20] T. Shang. Improving Performance of an Energy Efficient Hydraulic Circuit. PhD thesis, Department of Mechanical Engineering, University of Saskatchewan, Saskatoon, Canada, 2004.

[21] M. Linjama and M. Vilenius. Energy-efficient motion control of a digital hydraulic joint actuator. In Proceedings of the 6th JFPS International Symposium on Fluid Power, pages 640-645, Tsukuba, Japan, 2005. Japan Fluid Power System Society.

[22] Y. Jinghong, C. Zhaoneng, and L. Yuanzhang. The variation of oil effective bulk modulus with pressure in hydraulic systems. Journal of Dynamic Systems, Measurement, and Control, 116(1):146-150, 1994. 